\title{
Integrative analysis reveals key mRNA and long non-coding RNA interaction in idiopathic pulmonary arterial hypertension
}

\author{
Ruomeng Wang ${ }^{1}$, Qi Zhuang ${ }^{1}$, Jian Wang ${ }^{2}$, Menghui Yang ${ }^{2}$, Xueming Zhang ${ }^{2}$, Jieyan Shen ${ }^{1,2}$
}

\begin{abstract}
${ }^{1}$ Department of Cardiology, South Campus, Ren Ji Hospital, School of Medicine, Shanghai Jiao Tong University, 201100, Shanghai, China

2Department of Cardiology, Ren Ji Hospital, Shanghai Jiao Tong University School of Medicine, 200127, Shanghai, China
\end{abstract}

Submitted: 4 January 2019

Accepted: 16 July 2019

Arch Med Sci

DOI: https://doi.org/10.5114/aoms.2020.96074

Copyright $\odot 2020$ Termedia \& Banach

\begin{abstract}
Introduction: Idiopathic pulmonary arterial hypertension (IPAH) is a rare and sporadic form of pulmonary arterial hypertension (PAH), characterized by elevated pulmonary arterial resistance leading to right heart failure. However, molecular mechanisms of PAH development are still not completely understood.

Material and methods: In this study, we aimed to uncover key mRNAs and long non-coding RNA (IncRNAs), functional modules and pathways. Moreover, to detect the dysregulated pathway or biological function, we performed Gene Ontology (GO) and Kyoto Encyclopedia of Genes and Genomes (KEGG) enrichment analysis. PPI and co-expression networks were constructed to reveal the potential roles of PAH-related mRNAs and IncRNAs. Results: A total of 3,134 genes, including 945 up-regulated and 2,189 down-regulated genes, were identified to be differentially expressed in IPAH by differential expression analysis. We identified $T$ cell differentiation and the $T$ cell receptor signaling pathway as up-regulated in IPAH by using GO and KEGG analysis. Based on the PPI module analysis, we identified that the pro-inflammatory genes, such as OAS1, CXCL10, STAT1 and TLR4, were the hub genes in the PPI modules. To link the IncRNAs to the PPI modules, we calculated the Spearman correlation coefficient for IncRNA-DE-mRNA pairs to identify the modules with high correlation with each IncRNA.

Conclusions: Notably, 6 of these IncRNAs were associated with modules characterized by the NOD-like receptor signaling pathway and chemokine signaling pathway, suggesting that these IncRNAs may promote the occurrence of IPAH via participating in the pro-inflammatory pathways. In conclusion, our systematic analysis not only improved our understanding of the molecular mechanism, but also provided potential IncRNA biomarkers for further research.
\end{abstract}

Key words: long non-coding RNA, differential analysis, protein-protein interaction analysis, biomarker, idiopathic pulmonary arterial hypertension.

\section{Introduction}

Idiopathic pulmonary arterial hypertension (IPAH) is a rare and sporadic form of pulmonary arterial hypertension (PAH), which is characterized by elevated pulmonary arterial resistance leading to right heart failure.

\author{
Corresponding author: \\ Jieyan Shen \\ Department of Cardiology \\ South Campus \\ Ren Ji Hospital \\ School of Medicine \\ Shanghai Jiao Tong University \\ 2000 Yuejiang Road \\ 201100, Shanghai, China \\ E-mail: shenjieyan@renji.com
}


However, IPAH is not associated with an underlying condition or family history. Idiopathic pulmonary arterial hypertension has a mean age of $35 \pm 15$ years at diagnosis. Over the past decades, the proportion of older patients diagnosed with idiopathic PAH has been increased by the PAH epidemiology. In addition, IPAH is characterized by a high burden of comorbidities, such as obesity (30-38\%), systemic hypertension (27-42\%), type2 diabetes mellitus (14\%), and ischemic heart disease (10-12\%).

The symptoms of IPAH are not specific, commonly include dyspnea, weakness, and recurrent syncope, and can be easily confused with many other, more common conditions such as asthma, chronic obstructive pulmonary disease or sleep apnea; therefore, it is difficult to diagnose at an early stage. However, it is important to diagnose it earlier as it has been shown to be linked with better outcomes. Due to complexity, a series of tests, such as electrocardiogram (ECG), echocardiogram, ventilation/ perfusion scan (V/Q scan), pulmonary function tests, and right heart catheterization, are required to rule out other possible conditions, and confirm the diagnosis.

Pulmonary arterial hypertension patients could be treated by one of three relatively well-characterized pathways (nitric oxide, endothelin and prostacyclin), which are currently approved for PAH therapies. To elucidate the complex pathogenic mechanisms that underlie this disease, an increasing number of researchers are conducting intensive research efforts. For example, Yu et al. reported that TRPC channel overexpression may be partially responsible for the increased pulmonary arterial smooth muscle cell (PASMC) proliferation and pulmonary vascular medial hypertrophy in IPAH patients. Moreover, endothelin stimulates smooth muscle cell (SMC) proliferation via downstream signaling cascades involving endothelin type $A$ and type $B$ receptors. Furthermore, inflammation has a key role in human PAH as well as in experimental models of pulmonary arterial hypertension. However, the molecular mechanisms of PAH development are still not completely understood. In this study, we performed a systematic data analysis of key mRNAs and long non-coding RNA (IncRNAs) in peripheral blood mononuclear cells (PBMCS) of IPAH, which identified primarily dysregulated pathways, and highlighted key mRNAs and IncRNAs in PAH. The present study not only improved our understanding of the molecular mechanism, but also provided potential IncRNA biomarkers for further research. This way of bioinformation analysis has been widely applied in various disease fields.

\section{Material and methods}

\section{Data collection and format conversion}

Microarray-based gene expression data of 30 IPAH cases and 41 healthy donors were downloaded from the Gene Expression Omnibus (GEO, www.ncbi.nlm.nih.gov/geo/) database with accession number GSE33463. The gene expression data were median-normalized. The scaled intensity value was also log2-transformed.

\section{Differential expression analysis}

The gene expression data were used to identify differentially expression genes and IncRNAs by $t$-test. The differentially expressed genes/IncRNAs were identified at the threshold adjusted $p$-value $<0.05$. The up- or down-regulation status was determined based on the fold change for each gene/IncRNA.

\section{Gene Ontology and Kyoto Encyclopedia of Genes and Genomes enrichment analysis}

The GO and KEGG enrichment analysis was implemented in javaGSEA (version 3.0). The genes were pre-ranked based their differential expression levels ( $t$ statistics). 10,000 permutations were used to calculate the enrichment significance.

\section{Co-expression analysis of IncRNAs and PPI modules}

Gene set enrichment analysis was used to evaluate the over-representation of the highly correlated genes with a given IncRNA in the PPI modules. The gene set enrichment analysis (GSEA) was implemented in the fgsea package with prerank mode in $\mathrm{R}$ programming language. The genes were ranked based on their correlation with each IncRNA.

\section{Overrepresentation enrichment analysis}

Overrepresentation enrichment analysis (ORA) was also implemented using the WEB-based Gene Set Analysis Toolkit (WebGestalt). The Gene Ontology biological processes and KEGG pathways were selected as the functional database.

\section{Protein-protein interaction analysis}

The Search Tool for the Retrieval of Interacting Genes/Proteins (STRING) online software (https://string-db.org) was used to assess the interactions. The interactions of the proteins encoded by the differently expressed genes were searched using STRING online software. The PPI network was visualized using Cytoscape soft- 
ware (http://www.cytoscape.org). The Cytoscape MCODE plug-in (version 3.4.0) was applied to search for modules with highly connected nodes from the PPI network. The resulting network was subjected to module analyses with the Plugin MCODE with the following parameters: degree cut-off $\geq 3$, the nodes with edges $\geq 2$-core, and number of nodes $\geq 7$.

\section{Results}

\section{Identification of differentially expressed mRNAs and IncRNAs in idiopathic pulmonary arterial hypertension}

We first extracted the accession prefix from the RefSeq gene annotation to identify the IncRNArelated probes. The accession prefixes of NR and $X R$ were used to identify the IncRNAs. In total, 48,803 probes were used to quantify the transcriptome, of which 1,155 IncRNAs were identified based on the accession prefixes. To identify the differentially expressed genes, we conducted differential expression analysis by comparing IPAH patients with healthy donors. A total of 3,134 genes, including 945 up-regulated and 2,189 down-regulated genes, were identified to be differentially expressed between the two groups (FDR < 0.05, Figure 1 A). Particularly, 9 up-regulated and 109 down-regulated IncRNAs were identified (Figure $1 \mathrm{~B}$ ), the expression profiles of which showed a great difference between IPAH and healthy control groups.

\section{Gene Ontology and Kyoto Encyclopedia of Genes and Genomes enrichment analysis}

To identify IPAH-related GO terms and KEGG pathways, we conducted gene set enrichment analysis. The GO analysis identified 442 up- regulated and 75 down-regulated GO terms (FDR $<0.05$, Supplementary Table S1). The KEGG analysis also identified 24 up-regulated and 1 downregulated KEGG pathways (FDR $<0.05$, Supplementary Table S2).

Specifically, we identified establishment of protein localization to endoplasmic reticulum, protein localization to endoplasmic reticulum, translational initiation, nuclear transcribed mRNA catabolic process nonsense mediated decay, multi-organism metabolic process, RNA catabolic process, protein targeting to membrane, rRNA metabolic process, ribosome biogenesis, and alpha-beta $T$ cell differentiation as the top-ten most significantly up-regulated GO terms (Figure 2 A). In contrast, we also identified regulation of $T$ cell chemotaxis, gas transport, regulation of $\mathrm{T}$ cell migration, positive regulation of cellular extravasation, regulation of lymphocyte chemotaxis, oxygen trans- port, regulation of lymphocyte migration, regulation of monocyte chemotaxis, regulation of fever generation, and regulation of heat generation as the top-ten most significantly down-regulated GO terms (Figure 2 B). The up-regulated GO terms were primarily related to protein synthesis, RNA catabolic process and $T$ cell differentiation. Particularly, a T cell induced severe inflammatory response may promote the occurrence of $\mathrm{PAH}$. Moreover, the down-regulated GO terms could be summarized as the regulations of lymphocyte migration and chemotaxis, and fever or heat generation, suggesting that the down-regulation of lymphocyte mobility and fever or heat generation may be associated with PAH.

The top-ten most significant up-regulated KEGG pathways were ribosome, colorectal cancer, $T$ cell receptor signaling pathway, prostate cancer, acute myeloid leukemia, circadian rhythm, endometrial cancer, neurotrophin signaling pathway, spliceosome, and basal cell carcinoma (Figure 2 C). In accordance with the up-regulated GO terms, the $T$ cell receptor signaling pathway was also identified to be up-regulated by the KEGG pathway enrichment analysis. Although there was only one down-regulated KEGG pathway identified (Figure $2 \mathrm{D}$ ), the metabolism-related pathways accounted for $60 \%$ of the top-ten most significantly down-regulated KEGG pathways, indicating that the metabolism-related pathways played important roles in $\mathrm{PAH}$.

\section{Identification of modules in DEG-based PPI network}

To construct the PPI network, we mapped the up- and down-regulated genes to the PPI network curated by the STRING database, respectively. The PPI network for the upregulated genes contained 443 nodes and 1,386 edges (Supplementary Table S3). The nodes with the most connected adjacent nodes were deemed as hub nodes, and may have important functionality. We identified that OAS1 (2'-5'-oligoadenylate synthetase 1), GART (GAR transformylase), CXCL10 (C-X-C motif chemokine ligand 10), STAT1 (signal transducer and activator of transcription 1), and TLR4 (Toll like receptor 4) were the hub nodes in the up-regulated PPI network (connectivity degree > 30). It is worth noting that OAS1, CXCL10, STAT1 and TLR4 were the pro-inflammatory genes. The PPI network for the downregulated genes was constructed by 1,027 nodes and 6,625 edges, with hub nodes such as JUN and FOS (Supplementary Table S4). Notably, JUN and FOS formed the AP-1 transcription factor, which was a negative regulator of cell differentiation.

Furthermore, to identify the modules in the DEG-based PPI network, we performed a mod- 

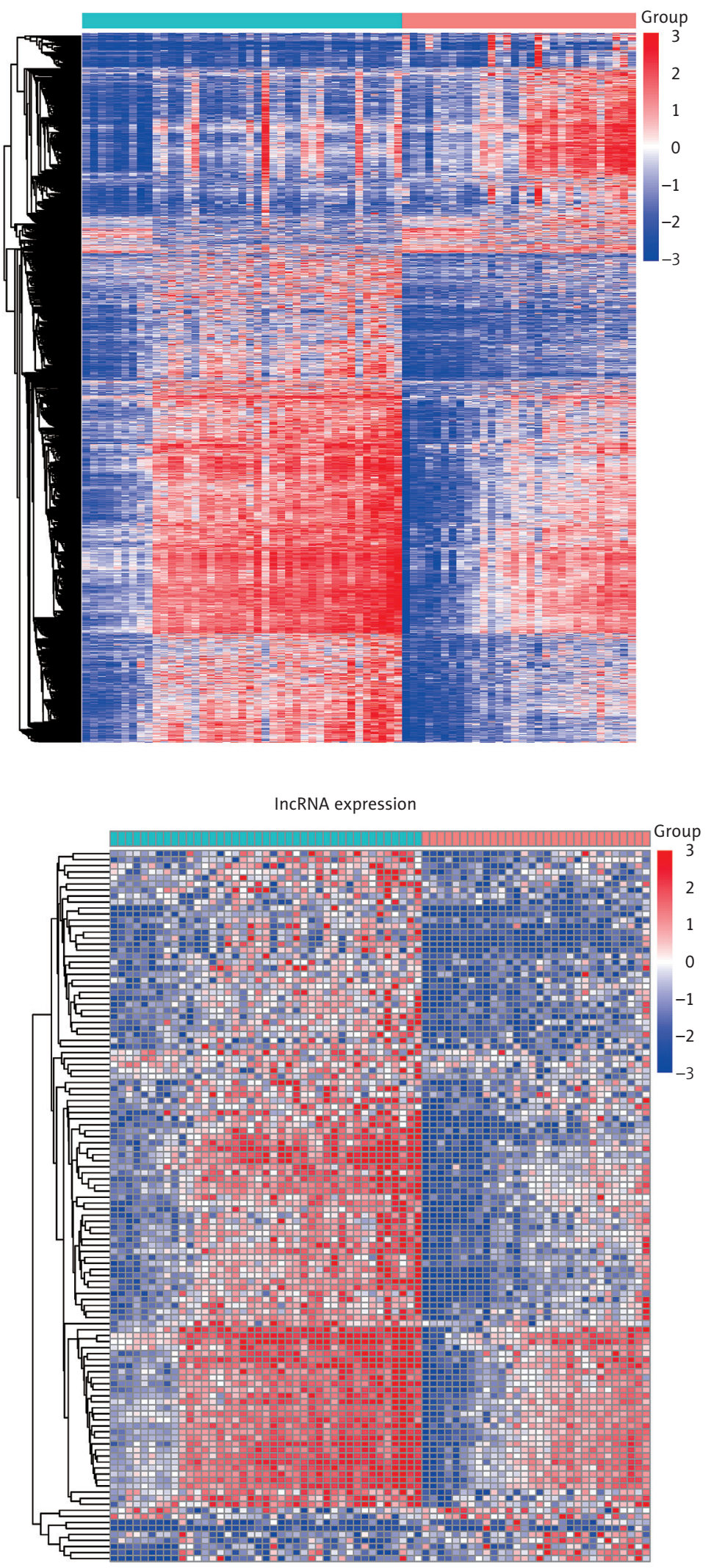

Figure 1. Differentially expressed genes between idiopathic pulmonary arterial hypertension (IPAH) cases and healthy controls. The two heatmaps display the mRNA (A) and long non-coding RNA (IncRNA) (B) expression profiles. The expression patterns of both mRNAs and IncRNAs are significantly different between the IPAH cases and healthy controls 


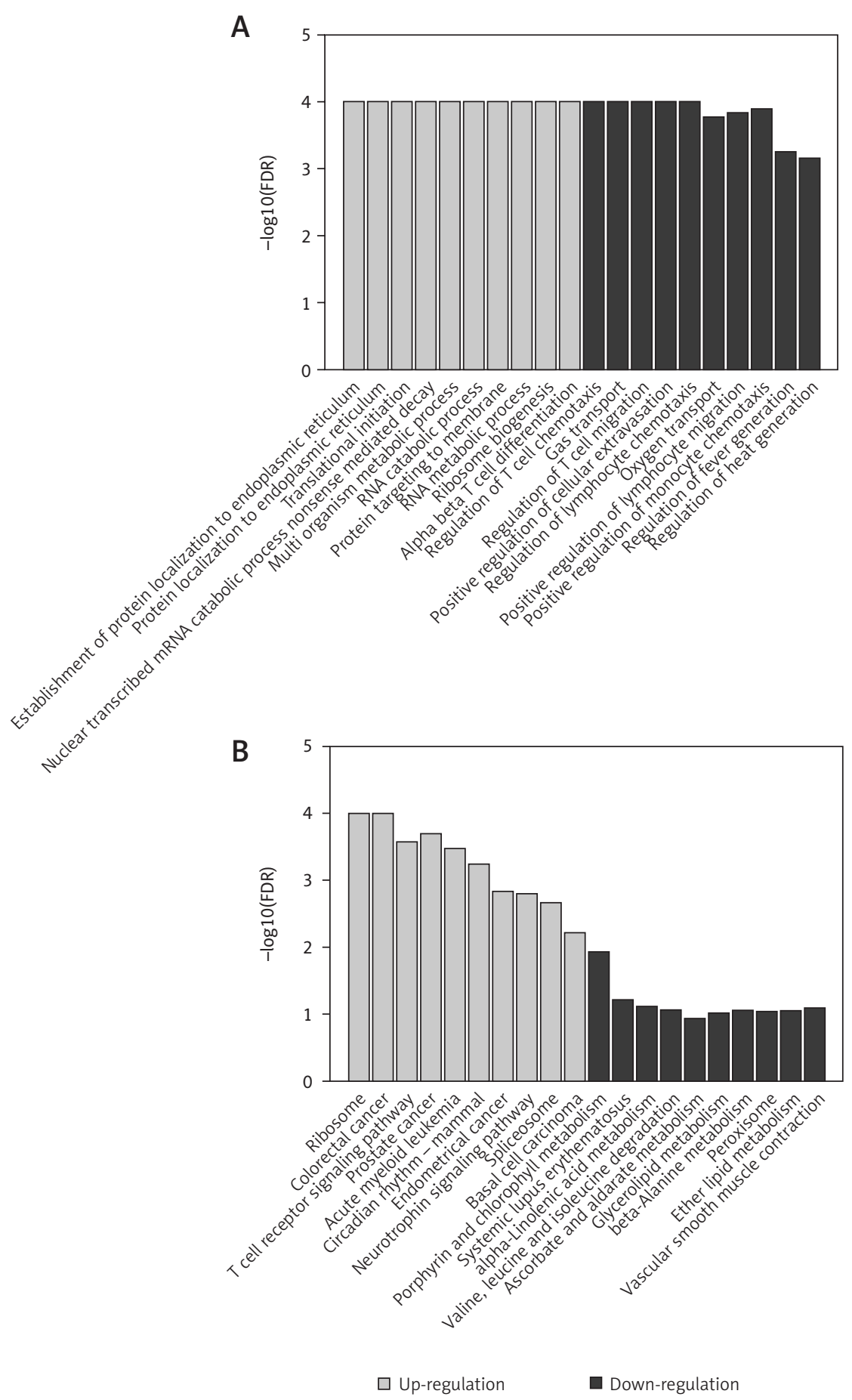

Figure 2. Gene set enrichment analysis (GSEA)-based Gene Ontology (GO) and Kyoto Encyclopedia of Genes and Genomes (KEGG) analysis. The enriched GO terms and KEGG pathways are presented in $\mathbf{A}$ and $\mathbf{B}$, respectively. The grey and black bars represent the GO terms or KEGG pathways enriched by up-regulated or down-regulated genes, respectively

ule analysis of the network using the MCODE plugin (degree cut-off $\geq 3$; the nodes with edges $\geq 2$-core; number of nodes $\geq 7$ ). We identified 7 up-regulated and 10 down-regulated modules in the PPI network, respectively. Particularly, the hub genes, such as OAS1, STAT1, TLR4, JUN and FOS, were also highlighted by the module analysis. In accordance with the GO and KEGG pathway enrichment analysis, the functional characterization of the PPI modules also revealed that pro-inflammatory pathways, such as the NOD-like receptor signaling pathway and chemokine signaling pathway, were highly enriched by the up-reg- 

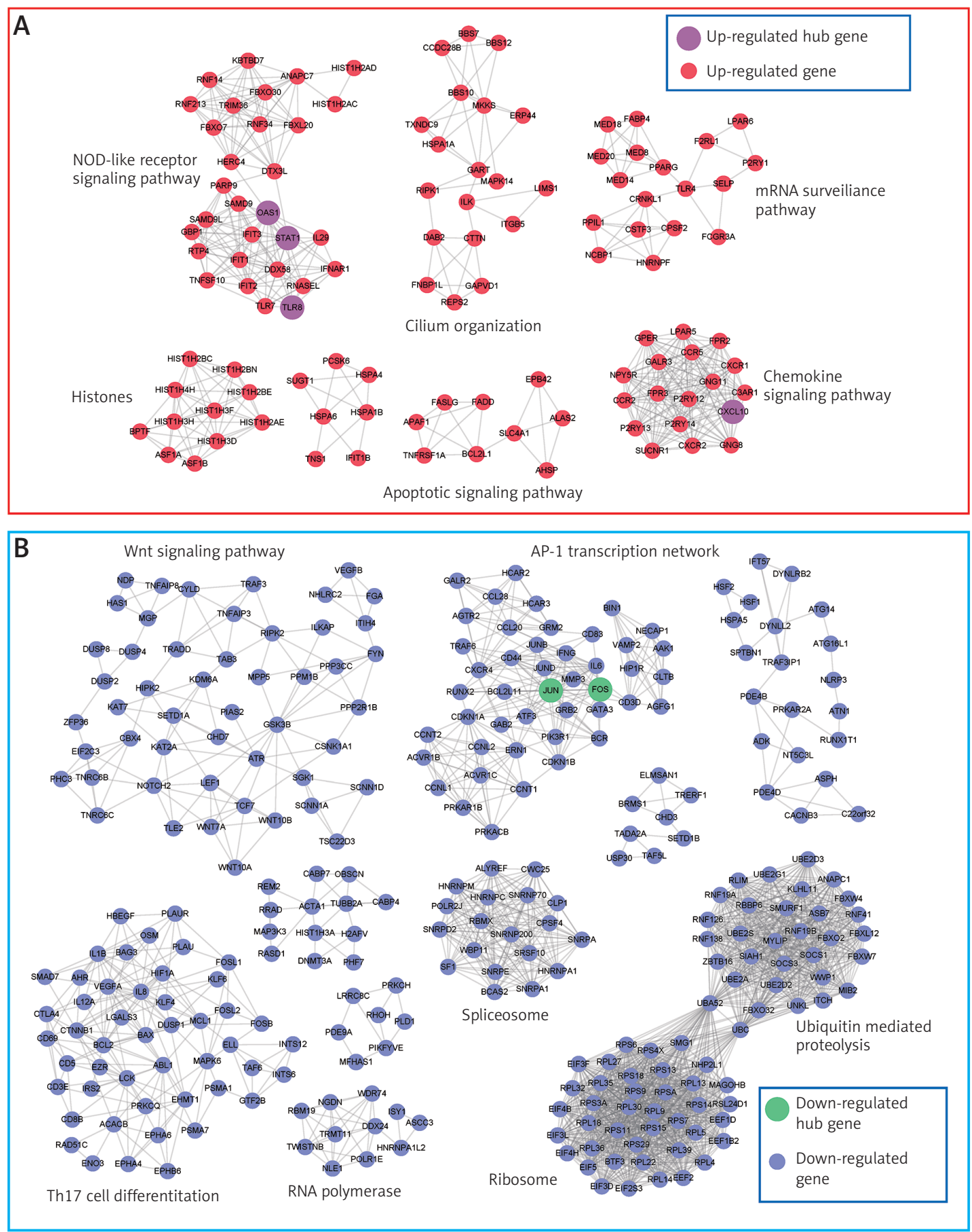

Figure 3. The up- and down-regulated PPI modules. The up- and down-regulated PPI modules are illustrated in A and B, respectively. The nodes with red and blue colors represent the up- and down-regulated genes. The PPI modules are characterized by Gene Ontology (GO) terms or Kyoto Encyclopedia of Genes and Genomes (KEGG) pathways based on overrepresentation enrichment analysis (ORA)

ulated modules based on over-representation enrichment analysis (ORA) (FDR $<0.05$, Figure 3 A). The down-regulated modules were significantly enriched in Wht signaling pathway, AP-1 transcription network, spliceosome, Th17 cell differentiation, RNA polymerase, ribosome, and ubiquitin mediated proteolysis (FDR $<0.05$, Figure $3 \mathrm{~B}$ ).
Co-expression analysis of IncRNAs and PPI modules

To link the differentially expressed IncRNAs with the PPI modules, we first calculated the correlation coefficient between each IncRNA and mRNA pair. Subsequently, GSEA was performed to evaluate the enrichment degree of the highly 
A
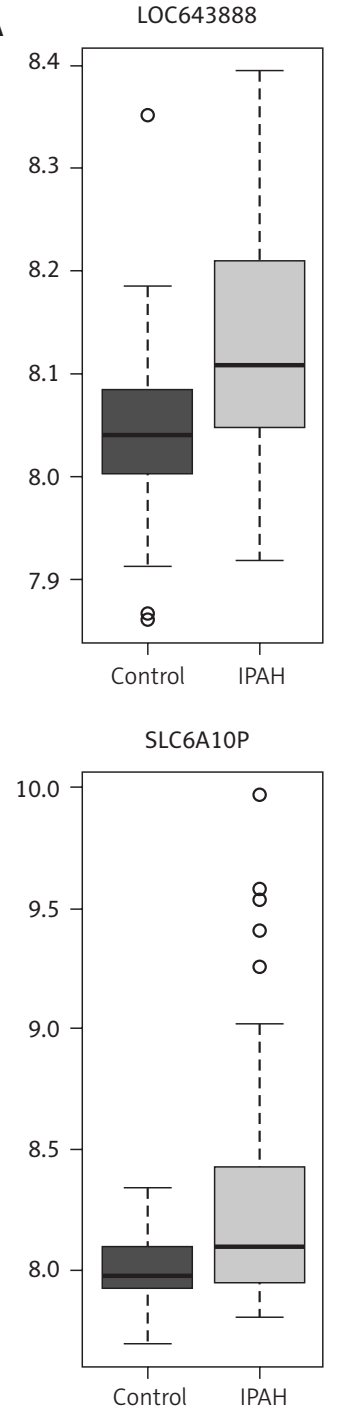

B

Chemokine signaling pathway

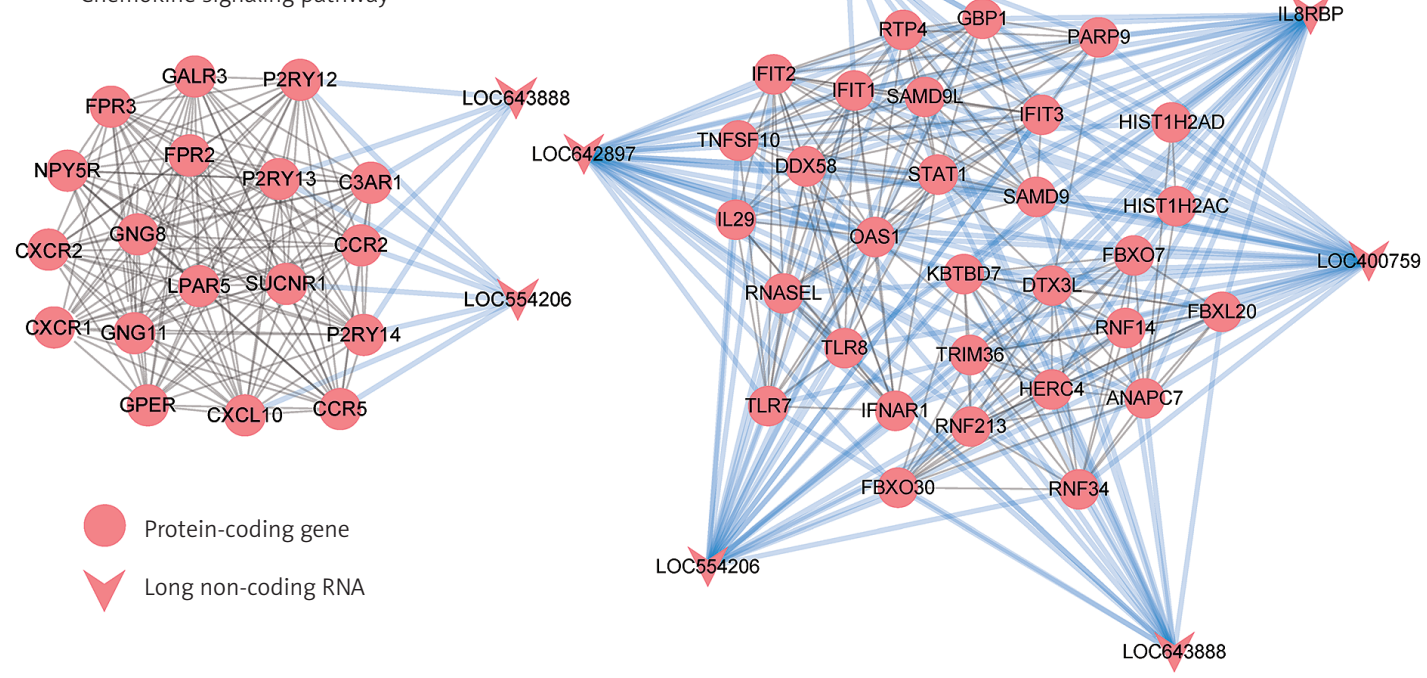

Figure 4. The expression patterns of seven key long non-coding RNAs (IncRNAs). The expression patterns of the seven IncRNAs in idiopathic pulmonary arterial hypertension (IPAH) and healthy controls are illustrated in A. The grey and black boxes indicate the IPAH and healthy control groups, respectively
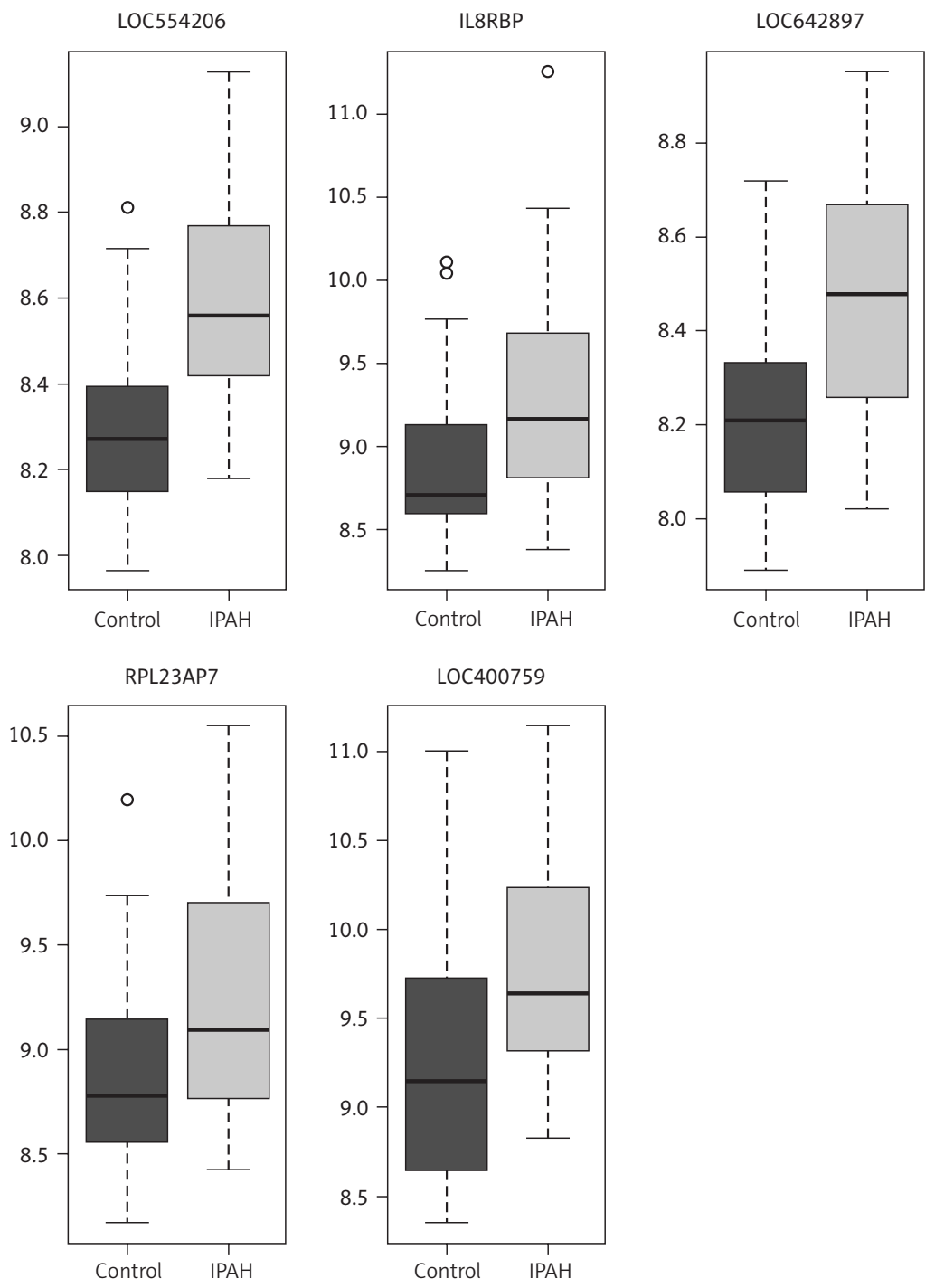

C

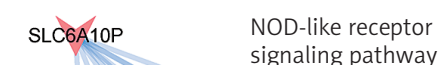

signaling pathway 


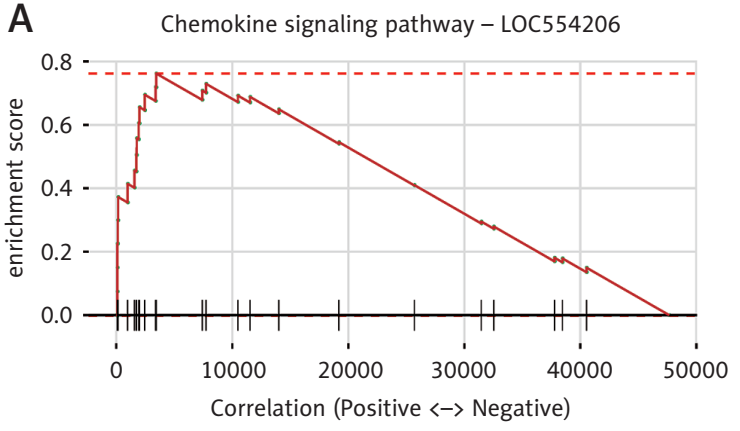

C

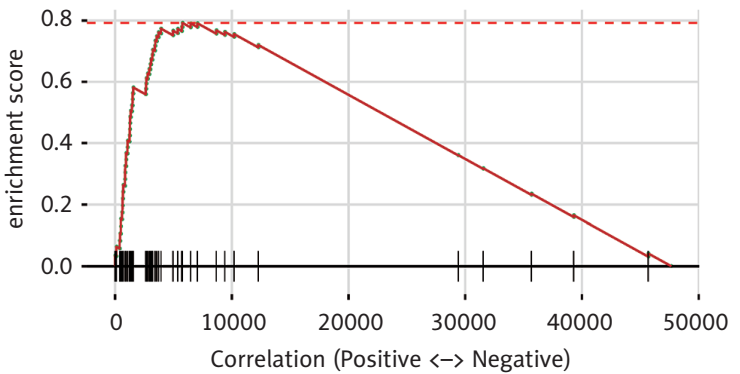

E

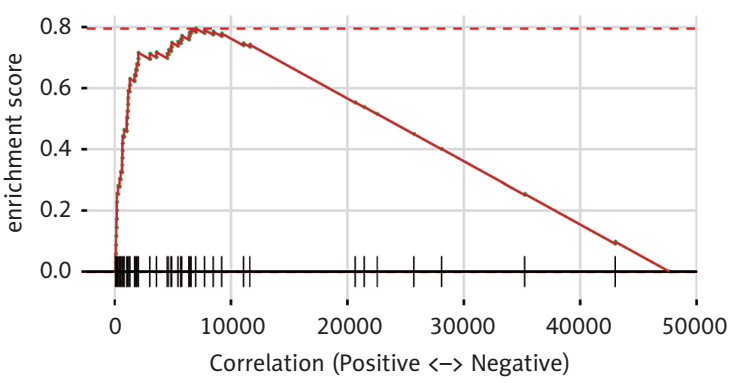

G

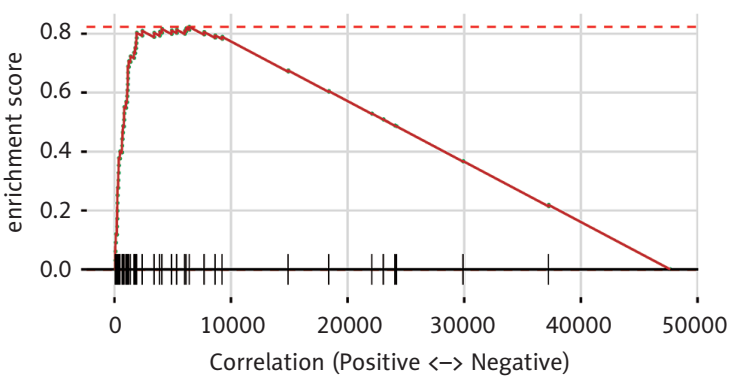

B Chemokine signaling pathway - LOC643888

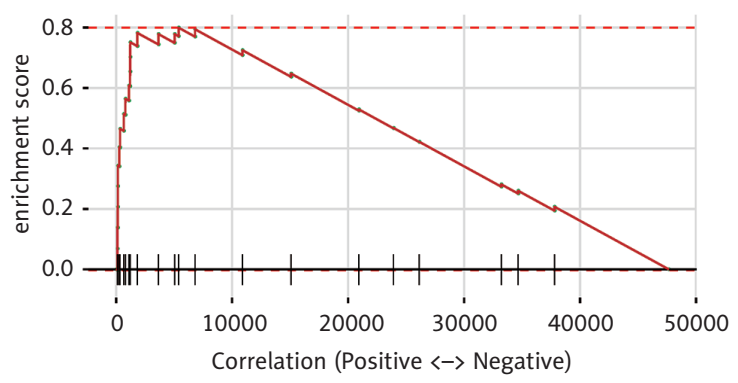

D NOD-like receptor signaling pathway - LOC400759

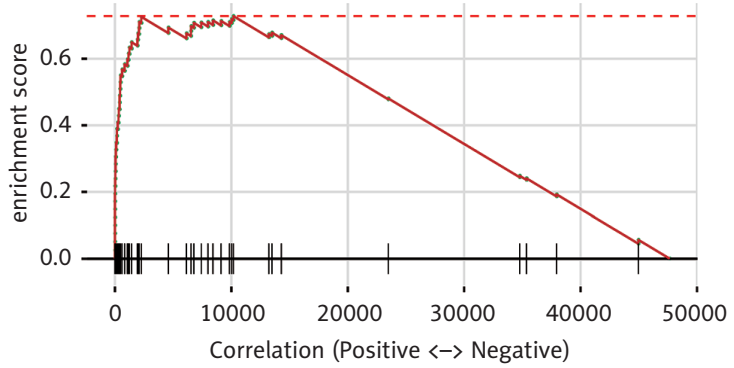

F NOD-like receptor signaling pathway - LOC642897

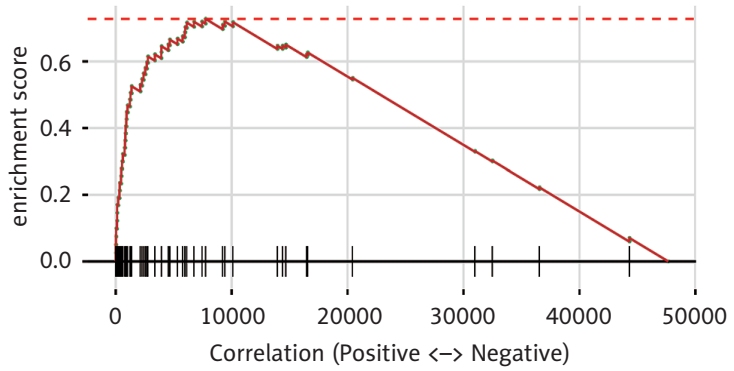

H NOD-like receptor signaling pathway - SLC6A10P

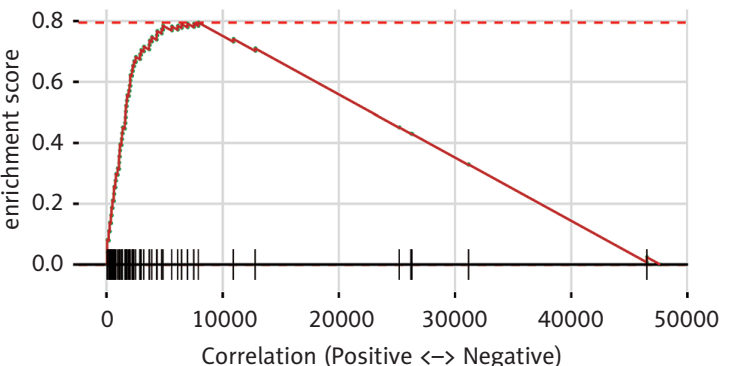

Figure 5. The gene set enrichment analysis of the pro-inflammatory pathway-related long non-coding RNA (InCRNA)-module pairs. Two IncRNAs, LOC554206 (A) and LOC643888 (B), are associated with modules characterized by the chemokine signaling pathway. Moreover, 6 other IncRNAs (IL8RBP, LOC400759, LOC554206, LOC642897, LOC643888 and SLC6A10P) are associated with NOD-like receptor signaling pathway (E-H)

correlated genes for each IncRNA in each module. We identified 18 pairs of IncRNA and PPI modules, including 7 IncRNAs and 4 PPI modules (adjusted $p$-value $<0.01)$. Interestingly, all 7 IncRNAs, i.e. LOC643888, LOC554206, IL8RBP, LOC642897, SLC6A10P, RPL23AP7 and LOC400759, were up-regulated in IPAH samples (Figure 4 A). The mRNA and IncRNA pairs were present in Fig- ures $4 \mathrm{~B}$ and $4 \mathrm{C}$, which showed that the IncRNAs had a high degree of connectivity in the network. The two IncRNAs, LOC643888 and LOC554206, were directly connected with purinergic receptors, such as P2RY12, P2RY13 and P2RY14, and C-C motif chemokine receptors, such as CCR2 and CCR5 (Figure 4 B). In contrast, among the seven IncRNAs, six were highly correlated with most 
of the genes involved in the other PPI module (Figure $4 \mathrm{C}$ ), indicating that these IncRNAs were closely associated with the PPI module.

Moreover, the GSEA based on the two PPI modules, which were characterized by pro-inflammatory pathways, the chemokine signaling pathway (Figures $5 \mathrm{~A}, \mathrm{~B}$ ) and the NOD-like receptor signaling pathway (Figures $5 \mathrm{C}-\mathrm{H}$ ), further demonstrated that these IncRNAs played critical roles in the pathogenesis of IPAH by participating in the pro-inflammatory pathways (adjusted $p$-value $<0.05$ ).

\section{Discussion}

Pulmonary arterial hypertension is a progressive disease of various origins, which has a poor prognosis and affects more than 100 million people worldwide. Idiopathic pulmonary arterial hypertension is defined as a rare subgroup of PAH, in which there is neither a family history of, nor an identified risk factor, for PAH.

In this study, we aimed to analyze the key mRNAs and IncRNAs in IPAH. To explore the molecular mechanism of IPAH, we collected gene expression datasets of PBMCs from IPAH cases and healthy donors. To identify the probes on the array corresponding to IncRNAs sequences, we identified a total of 1,155 IncRNAs based on the accession prefixes of NR and XR.

To investigate the biological differences between IPAH cases and healthy controls, we performed differential expression analysis of the gene expression data, and identified a total of 3,134 genes, including 945 up-regulated and 2,189 down-regulated genes, 118 of which were IncRNAs. Subsequently, the differentially expressed mRNAs were subjected to GO and KEGG enrichment analyses. The GO analysis identified 442 up-regulated and 75 down-regulated GO terms (FDR $<0.05$, Supplementary Table S1). The KEGG analysis also identified 24 up-regulated and 1 down-regulated KEGG pathways (FDR $<0.05$, Supplementary Table S2). The GO and KEGG enrichment analysis of the up-regulated genes revealed that the T cell receptor signaling pathway and $T$ cell differentiation played key roles in the occurrence of IPAH. Moreover, the down-regulated GO terms could be summarized as the regulations of lymphocyte migration and chemotaxis, and fever or heat generation, suggesting that the down-regulation of lymphocyte mobility and fever or heat generation may be closely associated with PAH.

To investigate the interactions between the proteins encoded by the DEGs, we mapped the DEGs to the PPI network. We observed that OAS1, CXCL10, STAT1 and TLR4 were key components in the modules of the up-regulated PPI network. Further functional characterization of these PPI modules revealed that pro-inflammatory pathways, such as the NOD-like receptor signaling pathway and chemokine signaling pathway, were highly enriched by the up-regulated modules based on over-representation enrichment analysis (ORA) (FDR < 0.05, Figure 3A).

To predict the potential functional roles of the differentially expressed IncRNAs, the Spearman correlation coefficient for IncRNA-DE-mRNA pairs was calculated to identify the modules with a high correlation with each IncRNA. Seven IncRNAs, i.e. LOC643888, LOC554206, IL8RBP, LOC642897, SLC6A10P,RPL23AP7 and LOC400759, showed a high correlation with PPI modules. Notably, 6 of these IncRNAs were associated with modules characterized by the NOD-like receptor signaling pathway and chemokine signaling pathway.

However, there are some limitations to this study. For example, the functional annotation of the IncRNAs by co-expression and PPI analysis can only suggest that the IncRNAs may participate in the subnetwork or pathway, while the specific regulatory relationships between the IncRNAs and protein-coding genes are still unknown, which requires more experiments to validate these interactions.

In conclusion, our integrative analysis revealed key mRNAs and IncRNAs, involved in IPAH. The results not only identified IPAH-related pro-inflammatory pathways such as the NOD-like receptor signaling pathway and chemokine signaling pathway, and IncRNAs, but also improved our understanding of the molecular mechanism for the occurrence of IPAH.

\section{Acknowledgments}

This work is supported by the National Natural Science Foundation of China (Grant Number 81570040), Health and Family planning Commission of Minhang Area (Grant No. 2016MW64).

\section{Conflict of interest}

The authors declare no conflict of interest.

\section{References}

1. Schermuly RT, Ghofrani HA, Wilkins MR, Grimminger F. Mechanisms of disease: pulmonary arterial hypertension. Nat Rev Cardiol 2011; 8: 443-55.

2. D'Alonzo GE, Barst RJ, Ayres SM, et al. Survival in patients with primary pulmonary hypertension. Results from a national prospective registry. Ann Intern Med 1991; 115: 343-9.

3. McGoon MD, Benza RL, Escribano-Subias P, et al. Pulmonary arterial hypertension: epidemiology and registries. J Am Coll Cardiol 2013; 62 (25 Suppl): D51-9.

4. Hoeper MM, Simon RGJ. The changing landscape of pulmonary arterial hypertension and implications for patient care. Eur Respir Rev 2014; 23: 450-7.

5. Ling Y, Johnson MK, Kiely DG, et al. Changing demographics, epidemiology, and survival of incident pulmonary arterial hypertension: results from the pulmonary 
hypertension registry of the United Kingdom and Ireland. Am J Respir Crit Care Med 2012; 186: 790-6.

6. Badesch DB, Raskob GE, Elliott CG, et al. Pulmonary arterial hypertension: baseline characteristics from the REVEAL Registry. Chest 2010; 137: 376-87.

7. Hoeper MM, Bogaard HJ, Condliffe R, et al. Definitions and diagnosis of pulmonary hypertension. J Am Coll Cardiol 2013; 62 (25 Suppl): D42-50.

8. Abman SH. New guidelines for managing pulmonary hypertension: what the pediatrician needs to know. Curr Opin Pediatr 2016; 28: 597-606.

9. Galiè N, Humbert M, Vachiery JL, et al. 2015 ESC/ERS Guidelines for the diagnosis and treatment of pulmonary hypertension: The Joint Task Force for the Diagnosis and Treatment of Pulmonary Hypertension of the European Society of Cardiology (ESC) and the European Respiratory Society (ERS): Endorsed by: Association for European Paediatric and Congenital Cardiology (AEPC), International Society for Heart and Lung Transplantation (ISHLT). Eur Respir J 2015; 46: 903-75.

10. Matsubara H, Ogawa A. Treatment of idiopathic/hereditary pulmonary arterial hypertension. J Cardiol 2014; 64: 243-9.

11. Yu Y, Fantozzi I, Remillard CV, et al. Enhanced expression of transient receptor potential channels in idiopathic pulmonary arterial hypertension. Proc Natl Acad Sci U S A 2004; 101: 13861-6.

12. Davie N, Haleen SJ, Upton PD, et al. ET(A) and ET(B) receptors modulate the proliferation of human pulmonary artery smooth muscle cells. Am I Respir Crit Care Med 2002; 165: 398-405.

13. Hassoun PM, Mouthon L, Barberà JA, et al. Inflammation, growth factors, and pulmonary vascular remodeling. J Am Coll Cardiol 2009; 54 (1 Suppl): S10-9.

14. Pullamsetti SS, Savai R, Janssen W, et al. Inflammation, immunological reaction and role of infection in pulmo nary hypertension. Clin Microbiol Infect 2011; 17: 7-14.

15. Kreymborg K, Uchida S, Gellert P, et al. Identification of right heart-enriched genes in a murine model of chronic outflow tract obstruction. J Mol Cell Cardiol 2010; 49: 598-605.

16. Sun LY, Cai ZY, Pu J, et al. 5-aminosalicylic acid attenuates monocrotaline-induced pulmonary arterial hypertension in rats by increasing the expression of Nur77. Inflammation 2017; 40: 806-17.

17. Hu X, Shen G, Lu X, Ding G, Shen L Identification of key proteins and IncRNAs in hypertrophic cardiomyopathy by integrated network analysis. Arch Med Sci 2019; 15: 484-97.

18. Jiang L, Hong L, Yang W, Zhao Y, Tan A, Li Y. Co-expression network analysis of the IncRNAs and mRNAs associated with cervical cancer progression. Arch Med Sci 2019; 15: 754-64.

19. Nisic F, Jovanovic N, Mavija M, et al. Vitreous concentrations of vascular endothelial growth factor as a potential biomarker for postoperative complications following pars plana vitrectomy. Arch Med Sci 2019; 15: 449-56.

20. Kang K, Huang YH, Li HP, Guo SM. Expression of UCA1 and MALAT1 long-chain non-coding RNAs in esophageal squamous cell carcinoma tissues is predictive of patient prognosis. Arch Med Sci 2018; 14: 752-9.

21. Cheadle C, Berger AE, Mathai SC, et al. Erythroid-specific transcriptional changes in PBMCs from pulmonary hypertension patients. PLoS One 2012; 7: e34951.

22. Subramanian A, Tamayo P, Mootha VK, et al. Gene set enrichment analysis: a knowledge-based approach for interpreting genome-wide expression profiles. Proc Natl Acad Sci U S A 2005; 102: 15545-50.
23. Ashburner M, Ball CA, Blake JA, et al. Gene ontology: tool for the unification of biology. The Gene Ontology Consortium. Nat Genet 2000; 25: 25-9.

24. Kanehisa M, Furumichi M, Tanabe M, Sato Y, Morishima K. KEGG: new perspectives on genomes, pathways, diseases and drugs. Nucleic Acids Res 2017; 45 (D1): D353-D61.

25. Szklarczyk D, Morris JH, Cook H, et al. The STRING database in 2017: quality-controlled protein-protein association networks, made broadly accessible. Nucleic Acids Res 2017; 45 (D1): D362-D8.

26. Bandettini WP, Kellman P, Mancini C, et al. MultiContrast Delayed Enhancement (MCODE) improves detection of subendocardial myocardial infarction by late gadolinium enhancement cardiovascular magnetic resonance: a clinical validation study. J Cardiovasc Magn Reson 2012; 14: 83.

27. Shaulian E, Karin M. AP-1 as a regulator of cell life and death. Nat Cell Biol 2002; 4: E131-6.

28. Peacock AJ, Murphy NF, McMurray JJV, Caballero L, Stewart S. An epidemiological study of pulmonary arterial hypertension. Eur Respir J 2007; 30: 104-9. 